\title{
一种比率型荧光探针用于成像活细胞中的过氧化氢
}

\author{
杨 敏 ${ }^{a}$ 夏丽丽 ${ }^{b}$ 周小琴 ${ }^{a}$ 贾程利 ${ }^{a}$ 吉 民*,a 王 鹏*,b \\ ( ${ }^{a}$ 东南大学生物科学与医学工程学院 南京 210009) \\ $\left({ }^{b}\right.$ 中国药科大学工学院 南京 210009)
}

\begin{abstract}
摘要 过氧化氢 $\left(\mathrm{H}_{2} \mathrm{O}_{2}\right)$ 以分子信号的形式在各种生理和病理过程中都起着重要作用, 因此快速、高效、灵敏地检测活 细胞或生物体中的 $\mathrm{H}_{2} \mathrm{O}_{2}$ 具有重要意义. 本研究设计了一种新型的 $\mathrm{H}_{2} \mathrm{O}_{2}$ 比率型苂光探针 HDP-1, 其对 $\mathrm{H}_{2} \mathrm{O}_{2}$ 表现出比率 荧光响应. 探针 HDP-1 在 $\mathrm{H}_{2} \mathrm{O}_{2}$ 检测方面具有选择性好、灵敏度高、细胞毒性低和反应速率快等优点, 同时, 该探针在 检测活细胞中外源性和内源性 $\mathrm{H}_{2} \mathrm{O}_{2}$ 都表现出了优异的性能.
\end{abstract}

关键词 比率型苂光探针; 外源; 内源; 过氧化氢; 活细胞成像

\section{A Ratiometric Fluorescent Probe for Imaging Hydrogen Peroxide in Living Cells}

\author{
Yang, $\operatorname{Min}^{a} \quad \mathrm{Xia} \mathrm{Lili}^{b} \quad$ Zhou, Xiaoqin $^{a} \quad \mathrm{Jia} \mathrm{Chengli}^{a} \quad \mathrm{Ji}, \mathrm{Min}^{*, a} \quad$ Wang, Peng ${ }^{*, b}$ \\ ( ${ }^{a}$ School of Biological Sciences and Medical Engineering, Southeast University, Nanjing 210009) \\ ( ${ }^{b}$ Department of Biomedical Engineering, School of Engineering, China Pharmaceutical University, Nanjing 210009)
}

\begin{abstract}
Hydrogen peroxide $\left(\mathrm{H}_{2} \mathrm{O}_{2}\right)$ plays an important role in various physiological and pathological processes in the form of molecular signals. Thus, it is of great significance to detect hydrogen peroxide in living cells or organisms quickly, efficiently and sensitively. In this study, a novel ratiometric fluorescent probe was designed to show the ratiometric fluorescence response to hydrogen peroxide. The probe showed the advantages of good selectivity, high sensitivity, low cytotoxicity and fast reaction rate in hydrogen peroxide detection. The probe also provided excellent performance in detecting both exogenous and endogenous hydrogen peroxide in living cells.
\end{abstract}

Keywords ratiometric fluorescent probe; exogenous; endogenous; hydrogen peroxide; living cell imaging

活性氧(ROS) 是一类高活性物质, 它们普遍存在于 生物体中, 在各种生理和病理过程中扮演着至关重要的 角色 ${ }^{[1]} \cdot \operatorname{ROS}$ 主要包括以下几种: 过氧化氢 $\left(\mathrm{H}_{2} \mathrm{O}_{2}\right)$ 、单线 态氧 $\left({ }^{1} \mathrm{O}_{2}\right)$ 、羟基自由基 $(\cdot \mathrm{OH})$ 、超氧阴离子 $\left(\cdot \mathrm{O}_{2}^{-}\right)$、过 氧化亚硝酰 $\left(\mathrm{ONOO}^{-}\right)$以及次氯酸 $\left(\mathrm{HOCl} / \mathrm{ClO}^{-}\right)^{[2]}$. 过氧 化氢 $\left(\mathrm{H}_{2} \mathrm{O}_{2}\right)$ 是活性氧中的强氧化剂，也是氧代谢的产物 之一. 研究表明, 过氧化氢在体内以信号分子的角色在 多种信号传递过程中起着关键作用, 在氧化应激过程中 同样起到重要作用 ${ }^{[3]}$. 越来越多的研究发现, 异常的细 胞会产生过量的过氧化氢, 而过量表达的过氧化氢会引 起多种疾病, 例如糖尿病 ${ }^{[4]}$ 、阿尔兹海默症 ${ }^{[5}$ 和癌症 ${ }^{[6]}$ 等, 因此, 快速、准确地检测活细胞中的 $\mathrm{H}_{2} \mathrm{O}_{2}$ 在疾病诊 断和预防方面具有重要意义.
传统的检测 $\mathrm{H}_{2} \mathrm{O}_{2}$ 的方法大都是侵入性检测, 会破 坏细胞和组织, 并且需要复杂的制备过程和操作过程, 这些缺陷限制了其广泛应用. 而荧光成像技术由于灵敏 性高、选择性高、时空分辨率高和生物相容性较好, 具 有实时监测和无创检测等优点, 在多种活性分子和离子 检测方面得到了大量应用研究 ${ }^{[7-12]}$. 近年来, 研究者们 成功开发了一系列检测 $\mathrm{H}_{2} \mathrm{O}_{2}$ 的有机小分子荧光探针, 初步实现了细胞和活体内 $\mathrm{H}_{2} \mathrm{O}_{2}$ 的实时监测, 建立了高 灵敏度、高选择性和实时成像的新方法 ${ }^{[13-17]}$. 但是已报 道的荧光探针都具有一定的局限性，比如检测限(DL)较 高( $\mathrm{DL}>100 \mathrm{nmol} / \mathrm{L})$ 、量子产率很低 $(0.02 \sim 0.04)$ 、反应 时间较长 $(t>1 \mathrm{~h})$ 等; 另外, 基于荧光强度变化的荧光探 针的生物学应用很容易受到激发/发射效率、探针的生物

* Corresponding authors. E-mail: wangpeng@cpu.edu.cn; 101010516@seu.edu.cn Received February 23, 2020; revised May 18, 2020; published online June 11, 2020.

Project supported by the General Program of National Natural Science Foundation of China (No. 81671745). 国家自然科学基金面上(No. 81671745)资助项目. 
分布及其局部微环境变化等的干扰. 以上条件都限制了 探针在 $\mathrm{H}_{2} \mathrm{O}_{2}$ 检测上的应用, 因此, 为了实现对活细胞中 $\mathrm{H}_{2} \mathrm{O}_{2}$ 更快速、高效、灵敏的检测, 比率型荧光探针的开 发得到了广泛关注 ${ }^{[18-22]}$. 比率型荧光探针依赖于分析物 引起的两个或更多个发射波段强度的变化提供内置校 正, 排除了背景荧光和外界干扰源的影响, 提供了准确 定量的分析. 目前, 比率型苂光探针已经成功实现了在 各种离子、生物分子以及细胞调亡等方面的监测应 用 $[22-25]$.

本工作组设计并合成了一种新型的有机小分子比 率型苂光探针 HDP-1, 用于特异性检测活细胞中外源和 内源性 $\mathrm{H}_{2} \mathrm{O}_{2}$ (Scheme 1), 以 1,8-菜内酰亚胺为原料合成 的阳离子化合物 1-乙基-2-甲基苯并 $[c d]$ 吲哚-1-氯化物 作为探针骨架, 对羟基苯甲醛作为连接基团, 芳香基硼 酸酯作为 $\mathrm{H}_{2} \mathrm{O}_{2}$ 的特异性识别基团. 4-澳甲基苯硼酸频哪 醇酯与对羟基苯甲醛以醚键的形式连接形成中间体, 中 间体再与 1-乙基-2-甲基苯并 $[c d$ 吲哚-1-氯化物经过脱 水缩合反应合成探针 HDP-1. 加入 $\mathrm{H}_{2} \mathrm{O}_{2}$ 后嗍酸酯基发 生裂解, 形成酚着基化合物 HDP-OH (Scheme 2), 使荧 光发射波长蓝移 (从 $618 \mathrm{~nm}$ 到 $485 \mathrm{~nm}$ ). 该探针实现了 对 $\mathrm{H}_{2} \mathrm{O}_{2}$ 快速、灵敏地特异性检测, 检测限为 $67 \mathrm{nmol} / \mathrm{L}$, 荧光量子产率约为 0.056 . 探针 HDP-1 还表现出较低的 细胞毒性, 并且能够成像活细胞中的外源和内源性 $\mathrm{H}_{2} \mathrm{O}_{2}$.

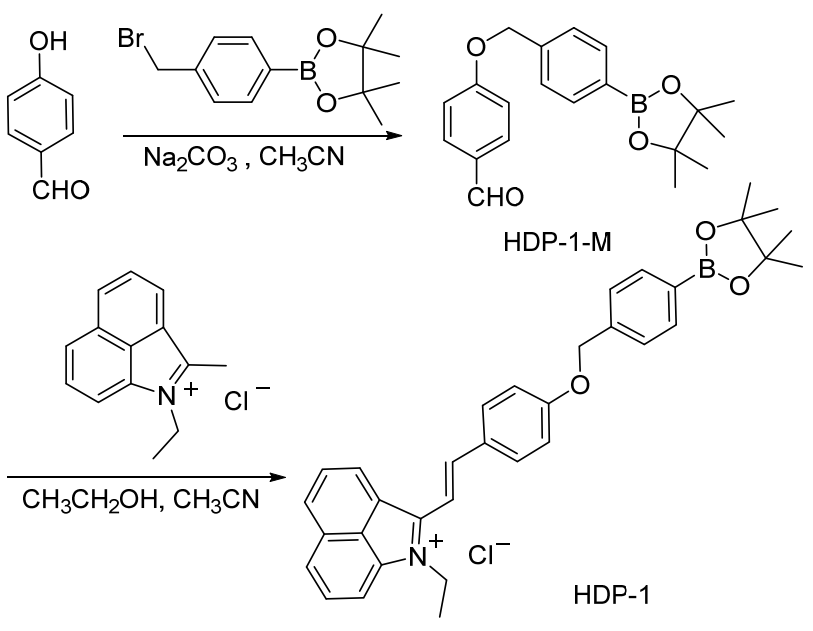

图式 1 探针 HDP-1 的合成路线

Scheme 1 Synthesis of probe HDP-1

\section{1 结果与讨论}

\section{1 探针 HDP-1 的设计与合成}

参考文献合成了 1-乙基-2-甲基苯并 $[c d$ ] 吲哚-1-氯 化物 ${ }^{[26-27]}$. 如 Scheme 1 所示, 以对着基苯甲醛和 4-澳甲 基苯嗍酸频哪醇酯为原料, 通过取代反应合成中间体

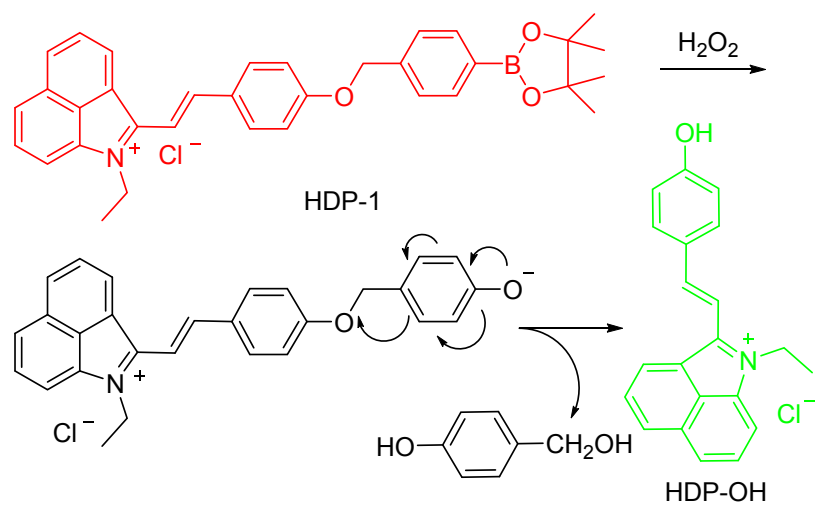

图式 2 HDP-1 与 $\mathrm{H}_{2} \mathrm{O}_{2}$ 反应的响应机制

Scheme 2 Proposed reaction mechanisms of HDP-1 toward $\mathrm{H}_{2} \mathrm{O}_{2}$

HDP-1-M, HDP-1-M 与已合成的 1-乙基-2-甲基苯并 $[c d$ ] 吲哚-1-氯化物通过脱水缩合反应生成探针 HDP-1. 1-乙 基-2-甲基苯并 $[c d]$ 吲哚-1-氯化物和 HDP-1 的结构都经 过 ${ }^{1} \mathrm{H} N M R,{ }^{13} \mathrm{C}$ NMR，质谱(MS)或高分辨质谱(HRMS) 确证.

\section{2 探针 HDP-1 的光谱性质}

为了研究探针 HDP-1 与 $\mathrm{H}_{2} \mathrm{O}_{2}$ 响应的光学性质, 将 $\mathrm{HDP}-1(10 \mu \mathrm{mol} / \mathrm{L})$ 与 $\mathrm{H}_{2} \mathrm{O}_{2}$ 在 PBS(磷酸盐缓冲液)溶液 [10\%二甲基亚砜(DMSO), $10 \mathrm{mmol} / \mathrm{L}, \mathrm{pH}=7.4]$ 中 $37{ }^{\circ} \mathrm{C}$ 孵育 $0.5 \mathrm{~h}$, 检测溶液的紫外可见光吸收光谱和荧光发 射光谱. 如图 1a 所示, 反应前探针 HDP-1 在 $500 \mathrm{~nm}$ 附 近有一个较高的吸收峰, 随着 $\mathrm{H}_{2} \mathrm{O}_{2}$ 浓度的升高, $500 \mathrm{~nm}$ 处的吸收峰明显降低, 并在 $620 \mathrm{~nm}$ 处出现一个新的最 大吸收峰, 且随着 $\mathrm{H}_{2} \mathrm{O}_{2}$ 浓度的增加而增强. 随后检测了 探针 HDP-1 与 $\mathrm{H}_{2} \mathrm{O}_{2}$ 反应前后的荧光光谱变化, 发现当 激发波长为 $420 \mathrm{~nm}$ 时, 未添加 $\mathrm{H}_{2} \mathrm{O}_{2}$ 的 HDP-1 在 $618 \mathrm{~nm}$ 处有较强的荧光发射, 而在 $485 \mathrm{~nm}$ 处只有微弱的荧光. 随着 $\mathrm{H}_{2} \mathrm{O}_{2}$ 浓度不断增加 $(0 \sim 500 \mu \mathrm{mol} / \mathrm{L}), 618 \mathrm{~nm}$ 处的 荧光逐渐减弱, $485 \mathrm{~nm}$ 处的荧光强度逐渐增强, 荧光发 射表现出较大的蓝移(133 nm), 使溶液从红色变成蓝色 (图 1b), 这表明探针 HDP-1 有望用于 $\mathrm{H}_{2} \mathrm{O}_{2}$ 的比率检测. 苂光发射比率 $\left(I_{485} / I_{618}\right)$ 从 0.15 增加到 12.02 , 增强了约 80 倍(图 1c). 发射比率 $\left(I_{485} / I_{618}\right)$ 在 $0 \sim 200 \mu \mathrm{mol} / \mathrm{L}$ 浓度范围 内表现出较好的线性相关性 $\left(R^{2}=0.9928\right)$ (图 1d), 经计 算得出检测限低至 $67 \mathrm{nmol} / \mathrm{L}$ (辅助材料中表 $\mathrm{S} 1$ ). 以上 结果表明, HDP-1 对生物体系内的 $\mathrm{H}_{2} \mathrm{O}_{2}$ 的响应具有较高 的灵敏度, 能够检测低浓度的 $\mathrm{H}_{2} \mathrm{O}_{2}$.

\section{3 动力学响应}

考察了 $\mathrm{HDP}-1$ 对 $\mathrm{H}_{2} \mathrm{O}_{2}$ 的时间响应. 如图 2a 所示, 在 PBS 溶液 $(10 \% \mathrm{DMSO}, 10 \mathrm{mmol} / \mathrm{L}, \mathrm{pH}=7.4)$ 中, HDP-1 在 $0 \sim 60 \mathrm{~min}$ 内苂光发射比 $\left(I_{485} / I_{618}\right)$ 都没有明显 

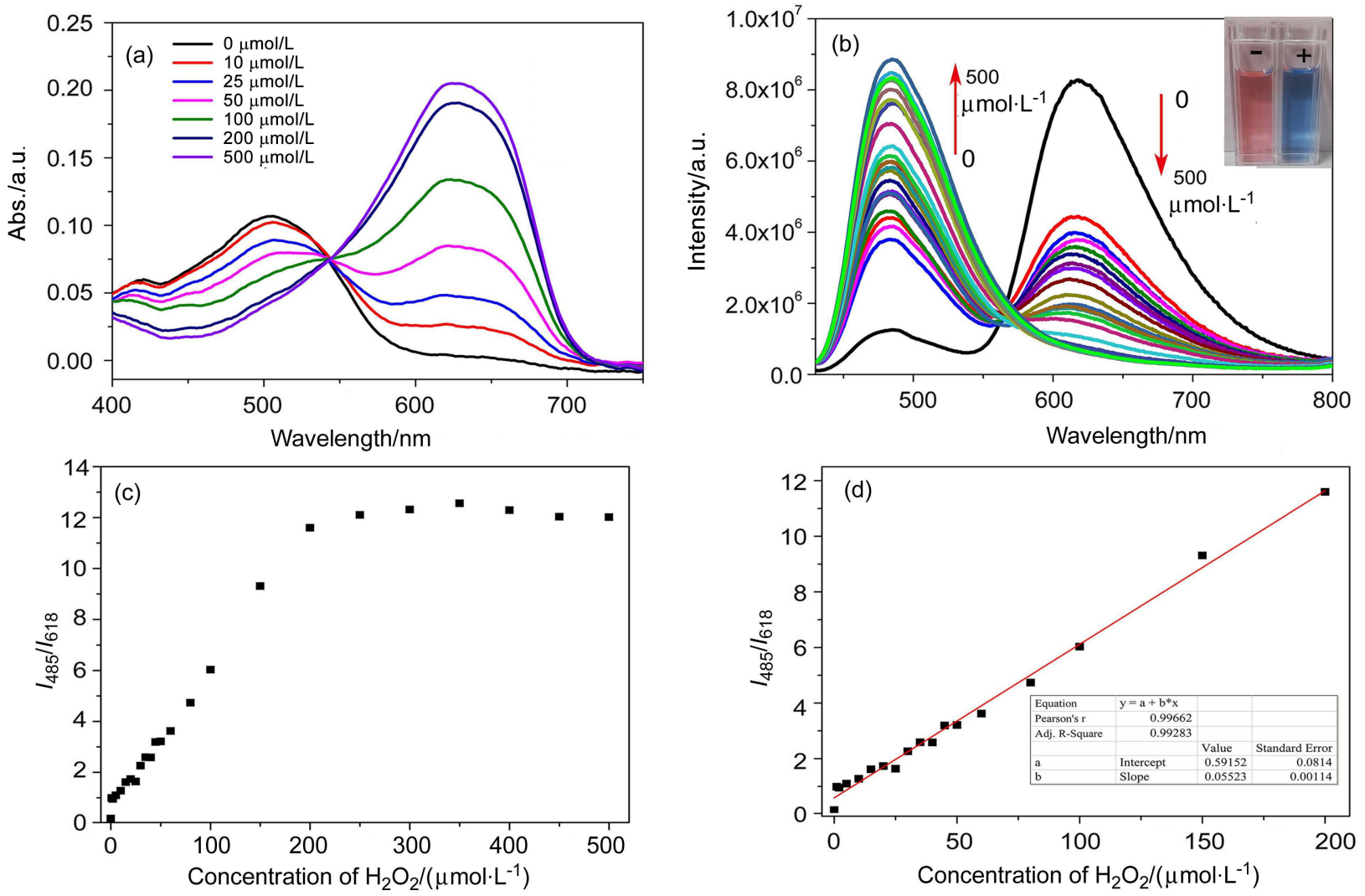

图 1 在 PBS 溶液( $10 \%$ DMSO, $10 \mathrm{mmol} / \mathrm{L}, \mathrm{pH}=7.4)$ 中, (a)探针 $\mathrm{HDP}-1(10 \mu \mathrm{mol} / \mathrm{L})$ 与不同浓度的 $\mathrm{H}_{2} \mathrm{O}_{2}(0 \sim 500 \mu \mathrm{mol} / \mathrm{L})$ 在 $37{ }^{\circ} \mathrm{C}$ 反 应 $0.5 \mathrm{~h}$ 后的紫外-可见光吸收光谱, (b)探针 HDP-1 $(10 \mu \mathrm{mol} / \mathrm{L})$ 与不同浓度的 $\mathrm{H}_{2} \mathrm{O}_{2}(0 \sim 500 \mu \mathrm{mol} / \mathrm{L})$ 在反应 $0.5 \mathrm{~h}$ 后的苂光发射光谱, (c)和(b)中的苂光发射比率 $I_{485} / I_{618}$, (d) $I_{485} / I_{618}$ 与 $\mathrm{H}_{2} \mathrm{O}_{2}$ 浓度 $(0 \sim 200 \mu \mathrm{mol} / \mathrm{L})$ 的线性相关性 $\left(\lambda_{\mathrm{ex}}=420 \mathrm{~nm}\right)$

Figure 1 (a) Absorption spectra of probe HDP-1 $(10 \mu \mathrm{mol} / \mathrm{L})$ in the absence/presence of $\mathrm{H}_{2} \mathrm{O}_{2}(0 \sim 500 \mu \mathrm{mol} / \mathrm{L})$ in $\mathrm{PBS}$ buffer $(\mathrm{pH}=$ 7.4, $10 \mathrm{mmol} / \mathrm{L}, 10 \% \mathrm{DMSO})$, (b) fluorescence spectra of probe HDP-1 $(10 \mu \mathrm{mol} / \mathrm{L})$ in response to $\mathrm{H}_{2} \mathrm{O}_{2}(0 \sim 500 \mu \mathrm{mol} / \mathrm{L})$ in PBS buffer $\left(\mathrm{pH}=7.4,10 \mathrm{mmol} / \mathrm{L}, 10 \%\right.$ DMSO), (c) the ratio of fluorescence intensity $\left(I_{485} / I_{618}\right)$ of probe HDP-1 $(10 \mu \mathrm{mol} / \mathrm{L})$ with the addition $\mathrm{H}_{2} \mathrm{O}_{2}$ $(0 \sim 500 \mu \mathrm{mol} / \mathrm{L})$ in PBS buffer $(\mathrm{pH}=7.4,10 \mathrm{mmol} / \mathrm{L}, 10 \% \mathrm{DMSO})$, and (d) the linear relationship between $I_{485} / I_{618}$ and the concentration of $\mathrm{H}_{2} \mathrm{O}_{2}(0 \sim 200 \mu \mathrm{mol} / \mathrm{L})\left(\lambda_{\mathrm{ex}}=420 \mathrm{~nm}\right)$

Insert: the color changes of the systems after adding $\mathrm{H}_{2} \mathrm{O}_{2}(500 \mu \mathrm{mol} / \mathrm{L})$ to the probe HDP-1 $(10 \mu \mathrm{mol} / \mathrm{L})$ under visible light. "—-" represents HDP-1 $(10 \mu \mathrm{mol} / \mathrm{L})$ without $\mathrm{H}_{2} \mathrm{O}_{2} ;$ “ " " represents HDP-1 $(10 \mu \mathrm{mol} / \mathrm{L})$ with $\mathrm{H}_{2} \mathrm{O}_{2}(500 \mu \mathrm{mol} / \mathrm{L})$
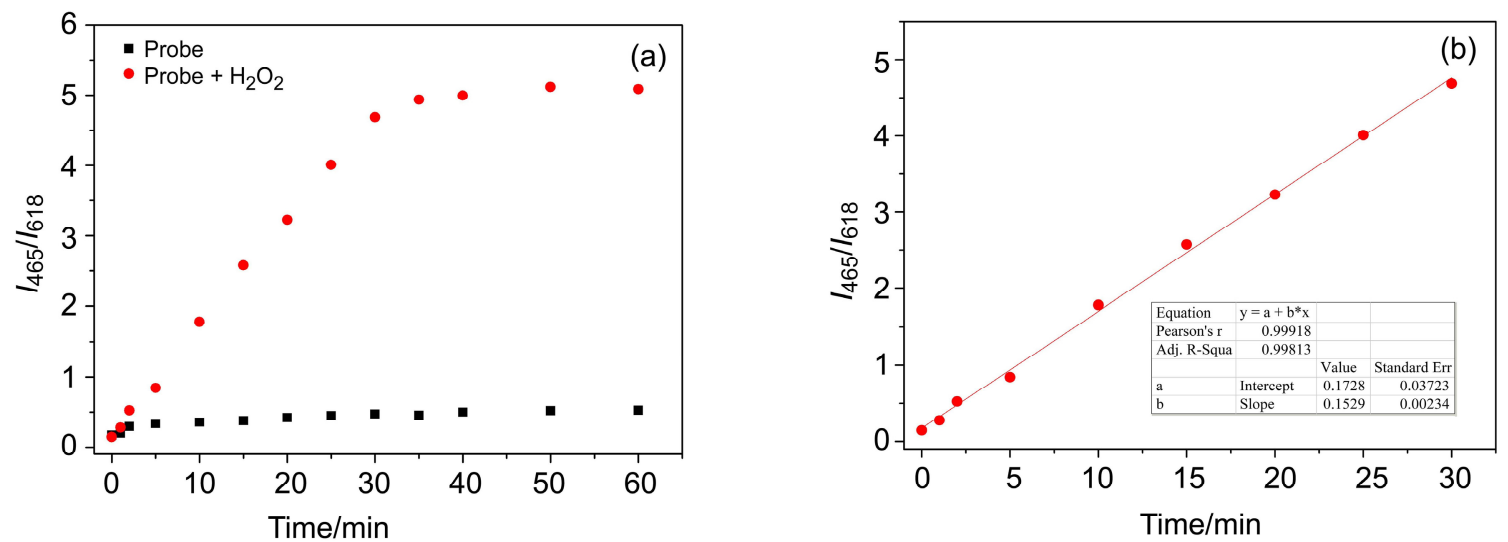

图 2 (a) HDP-1 $(10 \mu \mathrm{mol} / \mathrm{L})$ 与 $\mathrm{H}_{2} \mathrm{O}_{2}(200 \mu \mathrm{mol} / \mathrm{L})$ 在 $\mathrm{PBS}$ 缓冲液 $(\mathrm{pH}=7.4,10 \mathrm{mmol} / \mathrm{L}, 10 \% \mathrm{DMSO})$ 中反应后和仅存在 $\mathrm{HDP}-1(10$ $\mu \mathrm{mol} / \mathrm{L})$ 的苂光发射比 $I_{485} / I_{618}$ 随反应时间的变化，以及(b) $I_{485} / I_{618}$ 与反应时间 $(0 \sim 30 \mathrm{~min})$ 的线性关系 $\left(\lambda_{\mathrm{ex}}=420 \mathrm{~nm}\right)$

Figure 2 (a) Time-dependent fluorescence intensity ratio $\left(I_{485} / I_{618}\right)$ of HDP-1 $(10 \mu \mathrm{mol} / \mathrm{L})$ in the absence/presence of $\mathrm{H}_{2} \mathrm{O}_{2}(200$ $\mu \mathrm{mol} / \mathrm{L})$ in PBS buffer $(\mathrm{pH}=7.4,10 \mathrm{mmol} / \mathrm{L}, 10 \% \mathrm{DMSO})$, and (b) the linear relationship between $I_{485} / I_{618}$ and the reaction time with $\mathrm{H}_{2} \mathrm{O}_{2}(0 \sim 30 \mathrm{~min})\left(\lambda_{\mathrm{ex}}=420 \mathrm{~nm}\right)$ 
的变化, 表明了探针 HDP-1 的稳定性. 随着 $\mathrm{H}_{2} \mathrm{O}_{2}$ 浓度 升高, 探针 HDP-1 在 $485 \mathrm{~nm}$ 处的荧光强度逐渐增强, $618 \mathrm{~nm}$ 处的荧光强度逐渐减弱, HDP- 1 的发射比 $I_{485} / I_{618}$ 在 $30 \mathrm{~min}$ 左右趋于稳定(图 2a), 并且表现出良好的线性 相关 $\left(R^{2}=0.9981\right)$ (图 $\left.2 \mathrm{~b}\right)$. 从上述分析中可以看出, 探针 HDP-1 可以达到快速响应 $\mathrm{H}_{2} \mathrm{O}_{2}$ 的目的.

\section{$1.4 \mathrm{pH}$ 响应}

为了研究 $\mathrm{pH}$ 对探针 HDP-1 与 $\mathrm{H}_{2} \mathrm{O}_{2}$ 荧光响应的影 响, 在 $\mathrm{pH} 1 \sim 14$ 范围内检测了 $\mathrm{HDP}-1$ 对 $\mathrm{H}_{2} \mathrm{O}_{2}$ 的荧光变 化. 未添加 $\mathrm{H}_{2} \mathrm{O}_{2}, \mathrm{HDP}-1$ 的荧光发射比 $I_{485} / I_{618}$ 都很低 (低于 0.2 ), 并且在整个 $\mathrm{pH}$ 范围内没有明显的变化, 表 明 $\mathrm{HDP}-1$ 在不同 $\mathrm{pH}$ 下都是比较稳定的. 与 $\mathrm{H}_{2} \mathrm{O}_{2}(200$ $\mu \mathrm{mol} / \mathrm{L}$ )反应后, HDP-1 在 $\mathrm{pH} 6 \sim 8$ 范围内表现出非常显 著的比率响应, 并且在 $\mathrm{pH}=8$ 时达到最大值, 因此 HDP-1 可以用于检测生理条件下的 $\mathrm{H}_{2} \mathrm{O}_{2}$.

\section{5 选择性研究}

具有较好的选择性是荧光探针必不可少的性能之 一. 因此, 为了评价 HDP-1 对 $\mathrm{H}_{2} \mathrm{O}_{2}$ 的选择性, 检测了探 针 HDP-1 加入不同分析物 $\left(\mathrm{H}_{2} \mathrm{O}_{2} 、 \mathrm{ClO}^{-} 、 \cdot \mathrm{OH} 、 \cdot \mathrm{O}_{2}^{-}\right.$、 ${ }^{\circ}{ }^{t} \mathrm{Bu} 、 \mathrm{TBHP} 、 \mathrm{CN}^{-} 、 \mathrm{HSO}_{3}^{-} 、 \mathrm{Cu}^{2+} 、 \mathrm{Na}^{+} 、 \mathrm{Zn}^{2+} 、 \mathrm{Cys} 、$ $\mathrm{Hcy} 、 \mathrm{GSH}$ )后的苂光光谱响应. 如图 3 所示, 加入其他 分析物后, 探针 HDP-1 的荧光发射比 $I_{485} / I_{618}$ 与对照组 相比没有显著变化, 而与 $\mathrm{H}_{2} \mathrm{O}_{2}(200 \mu \mathrm{mol} / \mathrm{L})$ 反应后, HDP-1 的荧光发射比 $I_{485} / I_{618}$ 显著高于对照组和其他分 析物组. 另外, 在存在上述干扰分析物的情况下, 在 $\mathrm{HDP}-1$ 溶液中加入 $\mathrm{H}_{2} \mathrm{O}_{2}(200 \mu \mathrm{mol} / \mathrm{L})$ 进行竞争性实验. 结果表明, 干扰物的存在并不会影响探针 HDP-1 对

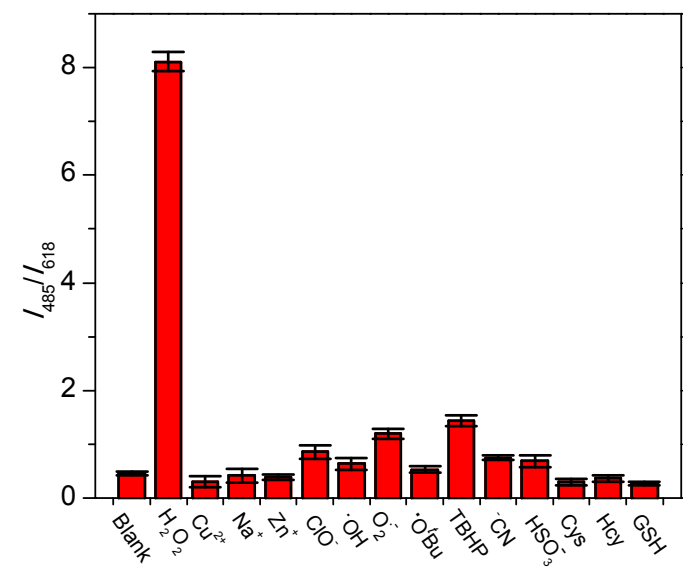

图 $3 \mathrm{HDP}-1(10 \mu \mathrm{mol} / \mathrm{L})$ 对不同检测物的荧光响应 $\left(I_{485} / I_{618}\right)$

Figure 3 Fluorescence intensity ratio $\left(I_{485} / I_{618}\right)$ of HDP-1 $(10$ $\mu \mathrm{mol} / \mathrm{L})$ to different detection substances

(1) Blank, (2) $\mathrm{H}_{2} \mathrm{O}_{2}(200 \mu \mathrm{mol} / \mathrm{L})$, (3) $\mathrm{Cu}^{2+}(1 \mathrm{mmol} / \mathrm{L})$, (4) $\mathrm{Na}^{+}(1$ $\mathrm{mmol} / \mathrm{L}),(5) \mathrm{Zn}^{2+}(1 \mathrm{mmol} / \mathrm{L})$, (6) $\mathrm{ClO}^{-}(500 \mu \mathrm{mol} / \mathrm{L})$, (7) $\bullet \mathrm{OH}(500$ $\mu \mathrm{mol} / \mathrm{L}),(8) \cdot \mathrm{O}_{2}^{-}(500 \mu \mathrm{mol} / \mathrm{L}),(9) \cdot \mathrm{O}^{t} \mathrm{Bu}(500 \mu \mathrm{mol} / \mathrm{L}),(10) \mathrm{TBHP}$ $(500 \mu \mathrm{mol} / \mathrm{L}),(11) \mathrm{CN}^{-}(500 \mu \mathrm{mol} / \mathrm{L}),(12) \mathrm{HSO}_{3}^{-}(500 \mu \mathrm{mol} / \mathrm{L}),(13)$ Cys $(1 \mathrm{mmol} / \mathrm{L}),(14) \mathrm{Hcy}(1 \mathrm{mmol} / \mathrm{L}),(15) \mathrm{GSH}(1 \mathrm{mmol} / \mathrm{L}) . \lambda_{\mathrm{ex}}=420$ $\mathrm{nm}$
$\mathrm{H}_{2} \mathrm{O}_{2}$ 的苂光响应能力. 这一结果证明了探针 HDP-1 对 $\mathrm{H}_{2} \mathrm{O}_{2}$ 具有较好的选择性，并且其性能不易被其他活性 物质干扰.

\subsection{HDP-1 的量子产率 $\left(\varphi_{\mathrm{u}}\right)$}

为了检测加入 $\mathrm{H}_{2} \mathrm{O}_{2}(200 \mu \mathrm{mol} / \mathrm{L})$ 后 HDP-1 的相对 苂光量子产率，选择了香豆素 153 为参比标准(在乙醇 中 $\left.\varphi_{\mathrm{s}}=0.38\right)$. 分别测得香豆素 153 和待测样品的吸光度 $(A<0.05)$ 和苂光光谱, 获得相关数据, 通过计算得出待 测样品的量子产率 $\varphi_{\mathrm{u}}=0.056$.

\section{$1.7 \mathrm{HDP}-1$ 的反应机理}

为了探究 HDP- 1 与 $\mathrm{H}_{2} \mathrm{O}_{2}$ 的响应机制, 对 HDP-1 与 $\mathrm{H}_{2} \mathrm{O}_{2}$ 反应后的产物的光谱性质和质谱进行了分析. 实 验发现, HDP- 1 与 $\mathrm{H}_{2} \mathrm{O}_{2}$ 反应后, 在 $620 \mathrm{~nm}$ 处有一个最 大吸收峰. 另外, 在 $420 \mathrm{~nm}$ 激发下, 反应体系在 $485 \mathrm{~nm}$ 附近也表现出了极强的苂光发射. 这与 HDP-OH 的光谱 性质相似. 根据以上现象提出了 $\mathrm{HDP}-1$ 与 $\mathrm{H}_{2} \mathrm{O}_{2}$ 的响应 机制猜想(Scheme 2). HDP-1 与 $\mathrm{H}_{2} \mathrm{O}_{2}$ 反应后, 芳香基嗍 酸酯基团裂解，探针发生连续的氧化反应和消除反应， 形成了酚羟基化合物 HDP-OH，其分子内电荷转移 (ICT)发生改变, $\pi$-电子共轭体系也降低, 从而使发射波 长从 $618 \mathrm{~nm}$ 蓝移到 $485 \mathrm{~nm}$, 蓝移程度较大 $(133 \mathrm{~nm})$. 通 过 HDP- 1 与 $\mathrm{H}_{2} \mathrm{O}_{2}$ 反应体系中分离的产物的质谱分析, 也印证了这一猜想 (HRMS calcd for $\mathrm{C}_{21} \mathrm{H}_{18} \mathrm{NO}^{+}$ 300.1383 , found 300.1383 ).

\subsection{HDP-1 在 HeLa 细胞中的荧光成像}

在苂光成像实验前评价了探针的细胞毒性. 结果表 明, 探针对人宫颈癌 HeLa 细胞、人乳腺癌 MCF-7 细胞 和人皮肤成纤维细胞 HSF 在较高浓度下 $(0 \sim 25 \mu \mathrm{mol} / \mathrm{L})$ 都没有表现出显著的细胞毒性, 这为 HDP-1 在活细胞 中的荧光成像提供了有力的支持. 随后采用苂光显微镜 探究了探针 HDP-1 在 HeLa 细胞中对外源和内源 $\mathrm{H}_{2} \mathrm{O}_{2}$ 的成像功能. 如图 4 所示, 在对照组中, 仅用探针 HDP-1 $(10 \mu \mathrm{mol} / \mathrm{L})$ 与 $\mathrm{HeLa}$ 细胞共培养, 在红色荧光通 道 $(570 \sim 625 \mathrm{~nm}$ ) 有较强的荧光发射，而在绿色荧光通 道 $(497 \sim 557 \mathrm{~nm}$ ) 只有很弱的荧光, 几乎观察不到. 实验 组 1 用脂多糖( $\mathrm{LPS}, 10 \mu \mathrm{g} / \mathrm{mL}$ ) 预处理 $0.5 \mathrm{~h}$ 后, 再用 HDP-1 $(10 \mu \mathrm{mol} / \mathrm{L})$ 与 HeLa 细胞共培养 $0.5 \mathrm{~h}$. 此时发现 红色荧光通道的荧光信号明显减弱, 绿色荧光通道的荧 光信号显著增强. 实验组 2 预先采用 $\mathrm{H}_{2} \mathrm{O}_{2}(100 \mu \mathrm{mol} / \mathrm{L})$ 处理 $0.5 \mathrm{~h}$, 再加入 HDP-1 $(10 \mu \mathrm{mol} / \mathrm{L})$ 与 $\mathrm{HeLa}$ 细胞共培 养 $0.5 \mathrm{~h}$. 与实验组 1 相似的是, 绿色荧光通道的荧光信 号能观察到显著的增强, 红色苂光通道的荧光信号则大 幅度减弱, 几乎观察不到. 研究结果表明, 探针 HDP-1 具有检测活细胞中外源和内源 $\mathrm{H}_{2} \mathrm{O}_{2}$ 的潜力. 


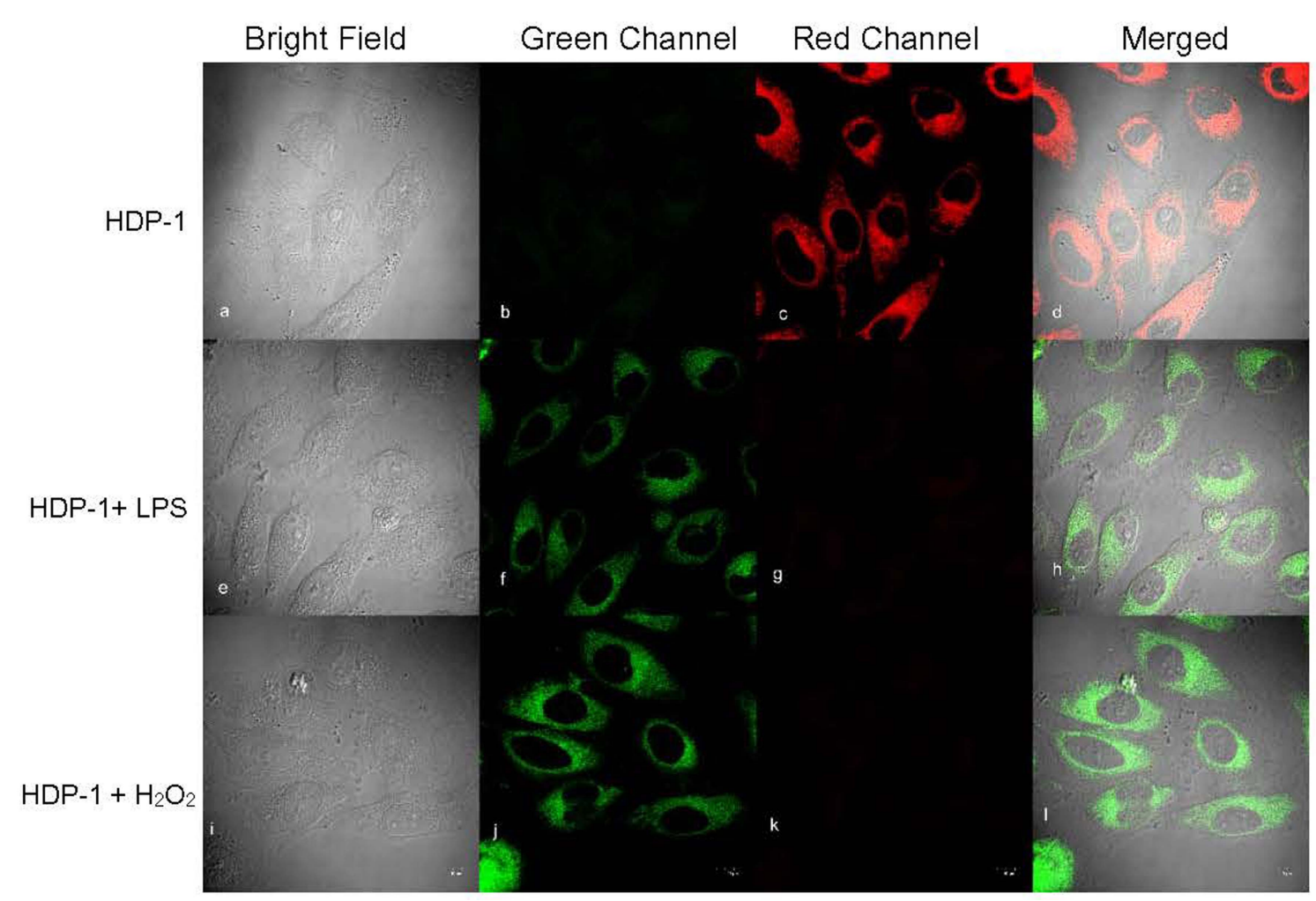

图 4 不同预处理条件下 HDP-1 $(10 \mu \mathrm{mol} / \mathrm{L})$ 在 $\mathrm{HeLa}$ 细胞中的共聚焦成像

Figure 4 Confocal images of HDP-1 $(10 \mu \mathrm{mol} / \mathrm{L})$ in HeLa cells under different pretreatment conditions

$(\mathrm{a} \sim \mathrm{d})$ Cells were co-cultured with probe HDP-1 $(10 \mu \mathrm{mol} / \mathrm{L}),(\mathrm{e} \sim \mathrm{h})$ cells were co-cultured with probe HDP-1 $(10 \mu \mathrm{mol} / \mathrm{L})$ for 30 min after pre-treatment with LPS $(10 \mu \mathrm{g} / \mathrm{mL})$, and $(\mathrm{i} \sim 1)$ cells co-cultured with probe HDP-1 $(10 \mu \mathrm{mol} / \mathrm{L})$ for $30 \mathrm{~min}$ after pre-treatment with $\mathrm{H}_{2} \mathrm{O}_{2}(100 \mu \mathrm{mol} / \mathrm{L})$. Green channel: $\lambda_{\mathrm{em}}=497 \sim 557 \mathrm{~nm}$. Red channel: $\lambda_{\mathrm{em}}=570 \sim 625 \mathrm{~nm} . \lambda_{\mathrm{ex}}=420 \mathrm{~nm}$. Scale bar: $10 \mu \mathrm{m}$

\section{2 结论}

综上所述, 设计了一种基于 ICT 效应的新型的比率 型荧光探针 $\mathrm{HDP}-1$, 用于 $\mathrm{H}_{2} \mathrm{O}_{2}$ 特异性检测. 该探针以硼 酸酯基为 $\mathrm{H}_{2} \mathrm{O}_{2}$ 识别基团, 能实现对 $\mathrm{H}_{2} \mathrm{O}_{2}$ 灵敏、快速的 特异性识别, 并且伴有肉眼可见的红色到蓝色的颜色变 化. 苂光发射波长蓝移达到 $133 \mathrm{~nm}$, 苂光发射比 $\left(I_{485} /\right.$ $I_{618}$ 增强达 80 倍, 检测限低至 $67 \mathrm{nmol} / \mathrm{L}$. 此外, 苂光成 像研究表明, 探针 HDP-1 可以有效地实现活细胞中的 内源和外源 $\mathrm{H}_{2} \mathrm{O}_{2}$ 的低毒性成像. 因此, 探针 HDP-1 在 活细胞中 $\mathrm{H}_{2} \mathrm{O}_{2}$ 的检测具有潜在的应用前景.

\section{3 实验部分}

\section{1 仪器与试剂}

所有的化学试剂和生物试剂均为市售化学纯或分 析纯产品, 除特别说明外, 均不需要进一步处理即可直 接使用. 基本必需培养基(MEM) 和磷酸盐缓冲溶液 (PBS) 购自 KeyGen Biotech(南京, 中国), 胎牛血清 (FBS)购自 Gibco (Grand Island, NY, USA). 采用 Quattro micro 三重四极杆质谱仪(中科牛津, 武汉, 中国)分析质 谱, Agilent 1100 LC/DAD/MSD 液质联用仪(Agilent, 美
国)进行高分辨质谱(HRMS)分析. ${ }^{1} \mathrm{H}$ NMR 和 ${ }^{13} \mathrm{C}$ NMR 在 Qone-WNMR-I-AS400 核磁共振波谱仪(中科牛津, 武汉, 中国)上进行. 使用 UV3600 UV-VIS-NIR 紫外分 光光度计 (岛津, 日本)记录吸收光谱. 在 FluoroMax-4 苂 光光谱仪(HORIBA, 美国)上收集发射光谱. 细胞活力 测定在多功能酶标仪(Thermo Fisher, MA, USA)上进行. 细胞荧光图像在 FV1000 激光共聚焦显微镜(Olympus, 日本)上获得.

\subsection{1-乙基-2-甲基苯并 $[c d]$ 䧄-1-氯化物的合成}

1,8-䒬内酰胺 $(8.03 \mathrm{~g}, 47.4 \mathrm{mmol}$ ) 溶于 $100 \mathrm{~mL} N, N$ 二甲基甲酰胺(DMF), 在冰浴条件下缓慢加入氢化钠 (3.528 g, $150 \mathrm{mmol})$. 体系冷却至室温后逐滴加入碘乙 烷 $(7.403 \mathrm{~g}, 50 \mathrm{mmol})$, 室温下搅拌 $0.5 \mathrm{~h}$. 硅胶柱层析分 离后, 旋干液体得到 $7.762 \mathrm{~g} \mathrm{1}$-乙基苯并 $[c d]$ 吲哚2(1H)-酮(黄色固体, 收率 83\%), 直接用于下一步反应.

将 1-乙基苯并 $[c d$ ] 吲哚-2(1H)-酮 $(1.972 \mathrm{~g}, 10 \mathrm{mmol})$ 和甲基氯化镁 $(15 \mathrm{~mL}, 45 \mathrm{mmol})$ 溶于无水四氢呋喃 (THF) $(40 \mathrm{~mL})$ 中, 在氮气保护下, $60{ }^{\circ} \mathrm{C}$ 加热回流至反 应完全. 将反应液冷却到室温, 加入少量水猝灭, 然后 用 $\mathrm{NaOH}$ 溶液 $(1 \mathrm{~mol} / \mathrm{L})$ 将反应液的 $\mathrm{pH}$ 调至碱性, 用乙 酸乙酯萃取 (30 $\mathrm{mL}$ 乙酸乙酯萃取 3 次); 向所得有机相 
中加入少量 $\mathrm{HCl}$ 溶液 $(1 \mathrm{~mol} / \mathrm{L})$, 将 $\mathrm{pH}$ 调至酸性, 用少量 水萃取 ( $20 \mathrm{~mL}$ 水萃取 2 次), 取水相; 在水相中加入 150 $\mathrm{mL}$ 丙酮并搅拌, 固体析出后进行抽滤, 并用丙酮淋洗

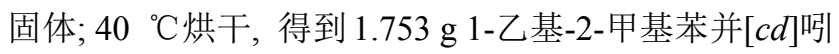
哚-1-氯化物 ${ }^{[28-30]}$, 收率 $76 \%$. m.p. $159 \sim 160{ }^{\circ} \mathrm{C} ;{ }^{1} \mathrm{H}$ NMR (400 MHz, MeOD) $\delta: 8.93(\mathrm{~d}, J=7.3 \mathrm{~Hz}, 1 \mathrm{H}), 8.79$ (d, $J=8.1 \mathrm{~Hz}, 1 \mathrm{H}), 8.47$ (d, $J=6.3 \mathrm{~Hz}, 1 \mathrm{H}), 8.46$ (d, $J=$ $7.1 \mathrm{~Hz}, 1 \mathrm{H}), 8.23 \sim 8.16(\mathrm{~m}, 1 \mathrm{H}), 8.07 \sim 8.02(\mathrm{~m}, 1 \mathrm{H})$, $4.83 \sim 4.79(\mathrm{~m}, 2 \mathrm{H}), 3.36 \sim 3.34(\mathrm{~m}, 3 \mathrm{H}), 1.72(\mathrm{t}, J=7.4$ $\mathrm{Hz}, 3 \mathrm{H}) ;{ }^{13} \mathrm{C}$ NMR (100 MHz, $\left.\mathrm{D}_{2} \mathrm{O}\right) \delta: 170.7$ (s), $138.3(\mathrm{~s})$, 137.3 (s), 134.3 (s), 130.8 (s), 130.5 (s), 129.4 (s), 128.1 (s), 127.7 (s), 121.4 (s), 120.4 (s), 42.3 (s), 14.5 (s), 12.2 (s); MS [ES-API] $m / z: 196.1[\mathrm{M}]^{+}$.

\section{3 探针 HDP-1 的合成}

将对羟基苯甲醛 $\left(0.122 \mathrm{~g}, 1 \mathrm{mmol}\right.$ )和 $\mathrm{Na}_{2} \mathrm{CO}_{3}(0.138$ $\mathrm{g}, 1 \mathrm{mmol}$ )溶于 $15 \mathrm{~mL}$ 无水乙腈中, 在 $60{ }^{\circ} \mathrm{C}$ 下搅拌 10 min. 然后加入 4-溴甲基苯嗍酸频哪醇酯( $0.328 \mathrm{~g}, 1.1$ $\mathrm{mmol})$ 和碘化钾 $(0.086 \mathrm{~g}, 0.5 \mathrm{mmol})$, 在氮气保护下 $60{ }^{\circ} \mathrm{C}$ 搅拌 $6 \mathrm{~h}$ 至反应完全. 抽滤后加入乙酸乙酯萃取 (30 mL 乙酸乙酯萃取 3 次) 并取有机相, 浓缩萃取液, 用 硅胶柱层析分离纯化 $[V$ (石油醚) $: V($ 乙酸乙酯 $)=6: 1]$ 得到 $0.315 \mathrm{~g}$ 中间体 HDP-1-M, 收率 93.2\%. ${ }^{1} \mathrm{H}$ NMR $\left(400 \mathrm{MHz}, \mathrm{CDCl}_{3}\right) \delta: 9.91(\mathrm{~s}, 1 \mathrm{H}), 7.87(\mathrm{~d}, J=4.2 \mathrm{~Hz}$, $2 \mathrm{H}), 7.85(\mathrm{~d}, J=5.0 \mathrm{~Hz}, 2 \mathrm{H}), 7.45(\mathrm{~d}, J=8.1 \mathrm{~Hz}, 2 \mathrm{H}), 7.09$ (d, $J=8.7 \mathrm{~Hz}, 2 \mathrm{H}), 5.20$ (s, 2H), 1.37 (s, 12H). HRMS calcd for $\mathrm{C}_{20} \mathrm{H}_{23} \mathrm{BO}_{4} 338.1689$, found 339.1690 .

将 HDP-1-M (0.236 g, $0.7 \mathrm{mmol})$ 和 1-乙基-2-甲基苯 并 $[c d$ 吲哚-1-氯化物 $(0.116 \mathrm{~g}, 0.5 \mathrm{mmol})$ 溶于乙醇/乙腈 $(V: V=9: 1)$ 的混合溶液中, 室温下氮气保护避光搅拌 至反应完全. 将混合液抽滤, 加入适量石油醚 $(20 \mathrm{~mL}$ 乙 醚(析出固体, 抽滤得到 $0.211 \mathrm{~g}$ 产物 HDP-1, 收率 76.7\%. m.p. $209 \sim 210{ }^{\circ} \mathrm{C} ;{ }^{1} \mathrm{H}$ NMR (400 MHz, $\left.\mathrm{CDCl}_{3}\right) \delta$ : $9.55(\mathrm{~s}, 1 \mathrm{H}), 8.81(\mathrm{~d}, J=8.3 \mathrm{~Hz}, 1 \mathrm{H}), 8.31(\mathrm{~s}, 2 \mathrm{H}), 8.23$ (d, $J=7.2 \mathrm{~Hz}, 1 \mathrm{H}), 8.14(\mathrm{~s}, 1 \mathrm{H}), 8.00(\mathrm{~d}, J=7.7 \mathrm{~Hz}, 1 \mathrm{H})$, $7.88 \sim 7.82(\mathrm{~m}, 4 \mathrm{H}), 7.65(\mathrm{t}, J=7.6 \mathrm{~Hz}, 1 \mathrm{H}), 7.33$ (d, $J=$ $8.0 \mathrm{~Hz}, 2 \mathrm{H}), 6.68$ (d, J=7.3 Hz,2H), 4.77 (s, 2H), 3.74 (q, $J=7.0 \mathrm{~Hz}, 2 \mathrm{H}), 1.57$ (s, 3H), 1.37 (s, 12H); ${ }^{13} \mathrm{C}$ NMR $(101$ $\left.\mathrm{MHz}, \mathrm{CDCl}_{3}\right) \delta: 163.1(\mathrm{~s}), 161.9(\mathrm{~s}), 155.0$ (s), 138.8 (s), 136.2 (s), 135.4 (s), 135.1 (s), $134.3(\mathrm{~s}), 131.6$ (s), 129.3 (s), $129.0(\mathrm{~s}), 128.5(\mathrm{~s}), 127.6(\mathrm{~s}), 126.4(\mathrm{~s}), 123.7$ (s), 115.4 (s), 112.7 (s), 83.9 (s), 69.8 (s), 24.9 (s), 15.7 (s). HRMS calcd for $\mathrm{C}_{34} \mathrm{H}_{45} \mathrm{BNO}_{3}$ 516.2704, found 516.2704.

\subsection{HDP-1 的光谱性质和苂光响应研究}

在 DMSO-PBS $(V: V=1: 9,10 \mathrm{mmol} / \mathrm{L}, \mathrm{pH}=7.4)$ 中, HDP-1 $(10 \mu \mathrm{mol} / \mathrm{L})$ 和不同浓度的 $\mathrm{H}_{2} \mathrm{O}_{2}(0 \sim 500$ $\mu \mathrm{mol} / \mathrm{L}$ )溶液在 $37^{\circ} \mathrm{C}$ 捊育 $30 \mathrm{~min}$, 然后检测其紫外-可 见光吸收光谱和苂光光谱. 在探针 HDP-1 对 $\mathrm{H}_{2} \mathrm{O}_{2}$ 的选 择性研究中, 其他 ROS 参照文献[31]方法制得母液. 将 HDP-1 $(10 \mu \mathrm{mol} / \mathrm{L})$ 与 $\mathrm{H}_{2} \mathrm{O}_{2}(200 \mu \mathrm{mol} / \mathrm{L})$ 、其他活性氧和 活性离子 $(500 \mu \mathrm{mol} / \mathrm{L})$ 、部分金属离子 $(1 \mathrm{mmol} / \mathrm{L})$ 、氨基 酸 $(1 \mathrm{mmol} / \mathrm{L})$ 在 DMSO-PBS $(V: V=1: 9,10 \mathrm{mmol} / \mathrm{L}$, $\mathrm{pH}=7.4)$ 中 $37{ }^{\circ} \mathrm{C}$ 孵育 $30 \mathrm{~min}$, 然后记录它们的荧光光 谱.

在探针 HDP-1 对 $\mathrm{H}_{2} \mathrm{O}_{2}$ 的时间响应研究中, 分别记 录 HDP-1 $(10 \mu \mathrm{mol} / \mathrm{L})$ 与/不与 $\mathrm{H}_{2} \mathrm{O}_{2}(500 \mu \mathrm{mol} / \mathrm{L})$ 孵育的 不同时间点 $(0,1,2,5,10,15,20,25,30,40,50,60 \mathrm{~min})$ 的荧光光谱. 在探针 HDP-1 的 $\mathrm{pH}$ 响应研究中, HDP-1 $(10 \mu \mathrm{mol} / \mathrm{L})$ 和 $\mathrm{H}_{2} \mathrm{O}_{2}(500 \mu \mathrm{mol} / \mathrm{L})$ 在不同 $\mathrm{pH}(1,2,3,4,5$, $6,7,8,9,10,11,12,13,14)$ 的 DMSO-PBS $(V: V=1: 9$, $10 \mathrm{mmol} / \mathrm{L}$ )中 $37{ }^{\circ} \mathrm{C}$ 捊育 $30 \mathrm{~min}$, 记录其苂光光谱. 所 有荧光光谱都在 $\lambda_{\mathrm{ex}}=420 \mathrm{~nm}$ 下收集溶液在 $430 \sim 800$ $\mathrm{nm}$ 的苂光发射, 激发和发射狭缝宽度都为 $5 \mathrm{~nm}$. HDP-1 在 DMSO 中制得 $1 \mathrm{mmol} / \mathrm{L}$ 母液再稀释使用.

\section{5 检测限}

对探针 HDP-1 (10 $\mu \mathrm{mol} / \mathrm{L})$ (空白组)在 $420 \mathrm{~nm}$ 激发 下的苂光强度进行 3 次测定, 获得空白组在 $618 \mathrm{~nm}$ 处苂 光强度的标准偏差. 根据荧光响应中 HDP-1 (10 $\mu \mathrm{mol} / \mathrm{L})$ 与 $\mathrm{H}_{2} \mathrm{O}_{2}(0 \sim 200 \mu \mathrm{mol} / \mathrm{L})$ 的线性相关计算检测限 (DL). 计算公式如下 ${ }^{[32]}$ :

$$
\mathrm{DL}=3 \sigma / k
$$

其中 $\sigma$ 是空白测量的标准偏差, $k$ 是 HDP-1 $(10 \mu \mathrm{mol} / \mathrm{L})$ 与 $\mathrm{H}_{2} \mathrm{O}_{2}(0 \sim 200 \mu \mathrm{mol} / \mathrm{L})$ 反应后 485 与 $618 \mathrm{~nm}$ 处苂光强 度的比值 $I_{485} / I_{618}$ 与不同 $\mathrm{H}_{2} \mathrm{O}_{2}$ 浓度之间的斜率.

\section{6 量子产率 $\left(\varphi_{\mathrm{u}}\right)$}

以香豆素-153 为参比标准, 用以下公式测定量子产 ${ }^{\text {率 }}{ }^{[33]}$ :

$$
\varphi_{\mathrm{u}}=\varphi_{\mathrm{s}} \cdot\left(I_{\mathrm{u}} / I_{\mathrm{s}}\right) \cdot\left(\mathrm{OD}_{\mathrm{s}} / \mathrm{OD}_{\mathrm{u}}\right) \cdot\left(N_{\mathrm{u}} / N_{\mathrm{s}}\right)^{2}
$$

其中 $\varphi$ 表示量子产率, $I$ 表示积分苂光强度, $\mathrm{OD}$ 表示 $\lambda_{\mathrm{ex}}$ 处的吸光度, $N$ 表示溶液折射率, $\mathrm{s}$ 和 $\mathrm{u}$ 分别表示参比品 和待测样品.

\subsection{HDP-1 对 $\mathrm{H}_{2} \mathrm{O}_{2}$ 的响应机制}

探针 HDP-1 $(10 \mu \mathrm{mol} / \mathrm{L})$ 与 $\mathrm{H}_{2} \mathrm{O}_{2}(500 \mu \mathrm{mol} / \mathrm{L})$ 在 DMSO-PBS $(V: V=1: 9,10 \mathrm{mmol} / \mathrm{L}, \mathrm{pH}=7.4)$ 中 $37{ }^{\circ} \mathrm{C}$ 卯育育 $30 \mathrm{~min}$, 然后加入少量乙酸乙酯 $(15 \mathrm{~mL}$ 乙 酸乙酯)萃取 3 次, 收集有机相, 旋转蒸发除去有机溶 剂后，对产物进行光学性质分析和质谱表征.

\section{8 细胞培养和 MTT 分析}

$\mathrm{HeLa}$ 细胞(人宫颈癌细胞)、MCF-7(人乳腺癌细胞), 
$\mathrm{HSF}$ (人皮肤成纤维细胞)均购自 KeyGen Biotech(南京, 中国). 细胞培养在添加了体积分数为 $10 \%$ 胎牛血清、 80 $\mathrm{U} / \mathrm{mL}$ 青霉素和 $0.08 \mathrm{mg} / \mathrm{mL}$ 链霉素的 MEM 中, 在体积 分数为 $5 \% \mathrm{CO}_{2}$ 培养箱中 $37{ }^{\circ} \mathrm{C}$ 培养 $24 \mathrm{~h}$.

采用噻唑蓝(MTT)法测定 HDP-1 对以上三种活细 胞的细胞毒性. 噻唑蓝购自 Solarbio(北京, 中国). HeLa 细胞、MCF-7 细胞和 HSF 细胞分别接种在 96 孔板中, 每 孔密度 $1 \times 10^{4}$, 在 $5 \% \mathrm{CO}_{2}$ 培养箱中 $37{ }^{\circ} \mathrm{C}$ 培养过夜. 将 不同浓度的 HDP-1 $(0,0.25,0.5,1,2.5,5,10,25,50,100$ $\mu \mathrm{mol} / \mathrm{L}$ )加入到细胞中继续培养 $24 \mathrm{~h}$, 然后将噻唑蓝(20 $\mu \mathrm{mol} / \mathrm{L}, 5 \mathrm{mg} / \mathrm{mL}$ )加入培养基继续孵育 $4 \mathrm{~h}$. 小心地去除 培养基, 每孔加入 $150 \mu \mathrm{L}$ 二甲基亚砜并摇 $10 \mathrm{~min}$ 以完 全溶解, 最后用多功能酶标仪 (Thermo Fisher, MA, USA)测定 $490 \mathrm{~nm}$ 处的吸光值，计算细胞存活率.

\section{9 成像实验}

苂光成像实验前一天, 在共聚焦培养皿中培养 HeLa 细胞, 共设置三组. 对照组只用 HDP-1 (10 $\mu \mathrm{mol} / \mathrm{L}$ )处理细胞. 实验组 1 用脂多糖( $\mathrm{LPS}, 10 \mu \mathrm{g} / \mathrm{mL}$ ) 预 处理 $0.5 \mathrm{~h}$ 后, 再与 HDP-1 (10 $\mu \mathrm{mol} / \mathrm{L})$ 共培养 $0.5 \mathrm{~h}$. 实 验组 2 用 $\mathrm{H}_{2} \mathrm{O}_{2}(100 \mu \mathrm{mol} / \mathrm{L})$ 预处理 $0.5 \mathrm{~h}$, 再与 HDP-1 $(10 \mu \mathrm{mol} / \mathrm{L})$ 共培养 $0.5 \mathrm{~h}$. 采用激光共聚焦显微镜进行细 胞成像观察.

辅助材料(Supporting Information) 中间体和终产物 的相关表征谱图, 实验方法以及部分苂光谱图和细胞实 验数据. 这些材料可以免费从本刊网站 (http://siocjournal.cn/)上下载.

\section{References}

[1] Wen, X.; Wu, J.; Wang, F.; Liu, B.; Huang, C.; Wei, Y. Free Radic. Biol. Med. 2013, 65, 402.

[2] Lou, Z.; Li, P.; Han, K. Acc. Chem. Res. 2015, 48, 1358.

[3] Balaban, R. S.; Nemoto, S.; Finkel, T. Cell 2005, 120, 483.

[4] Houstis, N.; Rosen, E. D.; Lander, E. S. Nature 2006, 440, 944.

[5] Teixeira, J. P.; de Castro, A. A.; Soares, F. V.; da Cunha, E. F. F.; Ramalho, T. C. Molecules 2019, 24, 4410.

[6] Ohshima, H.; Tatemichi, M.; Sawa, T. Arch. Biochem. Biophys. 2003, 417, 3 .

[7] Zhu, H.; Fan, J. L.; Wang, B. H.; Peng, X. J. Chem. Soc. Rev. 2015, 44, 4337.

[8] Chen, X. Q.; Wang, F.; Hyun, J. Y.; Wei, T. W.; Qiang, J.; Ren, X. T.; Shin, I.; Yoon, J. Chem. Soc. Rev. 2016, 45, 2976.

[9] Tian, Q.; Chen, S.; Chen, J.; Liu, R.; Wang, Y.; Yang, X.; Ye, Y. Chin. J. Org. Chem. 2019, 39, 2089 (in Chinese).
(田庆, 陈双虎, 陈景龙, 刘莣, 汪雨诗, 杨晓朋, 叶勇, 有机化 学, 2019, 39, 2089.)

[10] Chen, S.; Pang, C.; Chen, X.; Yan, Z.; Huang, S.; Li, X.; Zhong, Y.; Wang, Z. Chin. J. Org. Chem. 2019, 39, 1846 (in Chinese). (陈思鸿，庞楚明，陈孝云，严智浩，黄诗敏，李香弟，钟雅婷， 汪朝阳，有机化学, 2019, 39, 1846.)

[11] Huang, C.; Chen, H.; Li, F.; An, S. Chin. J. Org. Chem. 2019, 39, 2467 (in Chinese). (黄池宝，陈会，李福琴，安思雅，有机化学，2019, 39, 2467.)

[12] Wen, Y.; Huo, F.; Yin, C. Chin. Chem. Lett 2019, 30, 1834.

[13] Tang, L.; Tian, M.; Chen, H.; Yan, X.; Zhong, K.; Bian, Y. Dyes Pigm. 2018, 158, 482.

[14] Purdey, M. S.; McLennan, H. J.; Sutton-McDowall, M. L.; Drumm, D. W.; Zhang, X.; Capon, P. K.; Heng, S.; Thompson, J. G.; Abell, A. D. Sens. Actuators, B 2018, 262, 750.

[15] Shen, Y.; Zhang, X.; Zhang, Y.; Wu, Y.; Zhang, C.; Chen, Y.; Jin, J.; Li, H. Sens. Actuators B: Chem. 2018, 255, 42.

[16] Qiu, X.; Xin, C.; Qin, W.; Li, Z.; Zhang, D.; Zhang, G.; Peng, B.; Han, X.; Yu, C.; Li, L.; Huang, W. Talanta 2019, 199, 628.

[17] Zhang, J.; Shi, L.; Li, Z.; Li, D.; Tian, X.; Zhang, C. Analyst 2019, $144,36438$.

[18] Chen, Z.; Li, H.; Wei, J.; Xiao, Y.; Yu, H. Chin. J. Org. Chem. 2015, 35,789 (in Chinese). (陈忠林，李红玲，丰驾，肖义，于海波，有机化学， 2015，35, 789.)

[19] Gao, C.; Tian, Y.; Zhang, R.; Jing, J.; Zhang, X. Anal. Chem. 2017, 89,12945

[20] Hou, J.; Qian, M.; Zhao, H.; Li, Y.; Liao, Y.; Han, G.; Xu, Z.; Wang, F.; Song, Y.; Liu, Y. Anal. Chim. Acta 2018, 1024, 169.

[21] Wen, Y.; Liu, K.; Yang, H.; Li, Y.; Lan, H.; Liu, Y.; Zhang, X.; Yi, T. Anal. Chem. 2014, 86, 9970.

[22] Lee, M. H.; Kim, J. S.; Sessler, J. L. Chem. Soc. Rev. 2015, 44, 4185.

[23] Huang, Y.; Zhou, Q.; Feng, Y.; Zhang, W.; Fang, G.; Fang, M.; Chen, M.; Xu, C.; Meng, X. Chem. Commun. (Cambridge, U. K.) 2018, 54, 10495.

[24] Ning, P.; Hou, L.; Feng, Y.; Xu, G.; Bai, Y.; Yu, H.; Meng, X. Chem. Commun. 2019, 55, 1782.

[25] Ning, P.; Wang, W.; Chen, M.; Feng, Y.; Meng, X. Chin. Chem. Lett. 2017, 28, 1943.

[26] Xue, X.; Zhang, Y.; Liu, Z.; Song, M.; Xing, Y.; Xiang, Q.; Wang, Z.; Tu, Z.; Zhou, Y.; Ding, K.; Xu, Y. J. Med. Chem. 2016, 59, 1565 .

[27] Li, B.; Lu, L.; Zhao, M.; Lei, Z.; Zhang, F. Angew. Chem., Int. Ed. Engl. 2018, 57, 7483.

[28] Shi, Y.; Yuan, W.; Liu, Q.; Kong, M.; Li, Z.; Feng, W.; Hu, K.; Li, F. ACS Mater. Lett. 2019, 1, 418.

[29] Pilipchuk, N. V.; Piryatinski, Y. P.; Kachkovsky, G. O.; Slominskii, Y. L.; Kachkovsky, O. D. Dyes Pigm. 2007, 73, 353.

[30] Pilipchuk, N.; Piryatinski, Y.; Kachkovsky, G.; Slominskii, Y.; Kachkovsky, O. Dyes Pigm. 2007, 74, 47.

[31] Li, X.; Tao, R. R.; Hong, L. J.; Cheng, J.; Jiang, Q.; Lu, Y. M.; Liao, M. H.; Ye, W. F.; Lu, N. N.; Han, F.; Hu, Y. Z.; Hu, Y. H. J. Am. Chem. Soc. 2015, 137, 12296.

[32] Li, H.; Yao, Q.; Fan, J.; Du, J.; Wang, J.; Peng, X. Biosens. Bioelectron. 2017, 94, 536.

[33] Xiao, H.; Li, P.; Zhang, S.; Zhang, W.; Zhang, W.; Tang, B. Chem. Commun. (Cambridge, U. K.) 2016, 52, 12741. 